ARTÍCULOS ORIGINALES Rev Chil Salud Pública 2015; Vol 19 (1): 21-29

\section{LA DISTRIBUCIÓN GRATUITA DE CONDONES A TRABAJADORAS SEXUALES EN ECUADOR: EFECTOS EN EL COMPORTAMIENTO DE COMPRA}

\author{
FreE DISTRIBUTION OF CONDOMS TO FEMALE SEX WORKERS IN \\ ECUADOR: EFFECTS ON PURCHASE BehaVIOR
}

\section{RESUMEN}

Los condones son considerados un método eficaz para evitar la propagación de infecciones de transmisión sexual. En Ecuador, un factor común en las politicas públicas de salud sexual es la entrega gratuita de condones a trabajadoras sexuales (TS). Esto ocurre junto con el asesoramiento sobre otras medidas preventivas y la importancia de las pruebas de detección del VIH. Este estudio busca determinar si la distribución gratuita de condones afecta la compra de los mismos y su uso consecuente. A través de una encuesta aplicada a 2.566 trabajadoras sexuales en ocho ciudades del Ecuador, se analiza el comportamiento de compra de condones. Los datos corresponden a las encuestas realizadas por el Proyecto de Prevención de Fronteras (PPF) en Ecuador en 2006. Dado que el acceso a las políticas públicas y a los condones gratuitos no es aleatorio, existen riesgos de sesgos de selección muestral. Para ello utilizamos un procedimiento de estimación Heckman en dos etapas. En la primera se analiza la probabilidad de comprar condones, y en la segun$d a$, la cantidad de condones comprados. Se encuentra que por cada condón recibido gratuitamente, la TS compra uno más. Este resultado muestra la efectividad de las politicas de distribución cuando son acompañadas de componentes de asesoramiento e información.

Palabras clave: Procedimiento de Heckman, sesgo de selección muestral, Proyecto de Prevención en Fronteras, acceso, trabajadoras sexuales, condones.

\begin{abstract}
Condoms are considered an effective method to prevent the spread of sexually transmitted infections. In Ecuador, a common factor in sexual bealth public policy is the provision of free condoms to female sex workers. This occurs together with advice on other preventive measures and the importance of HIV screening. The present study seeks to determine whether
\end{abstract}

Recibido 19 de mayo 2014; aceptado 11 de diciembre 2014

Financiamiento: Corporación Kimirina 
the distribution of free condoms affects their purchase and subsequent use. A survey to assess condom purchase behavior was completed by 2566 female sexual workers (FSW) in eight cities in Ecuador. The Frontiers Prevention Project (FPP) conducted surveys during 2006. There is a risk of sample selection bias as access to public policy and free condoms is not random: we therefore used a Heckman estimation procedure in two stages. The probability of purchasing condoms and the quantity of condoms purchased were analyzed. We find that for each condom received for free, FSW purchased one more. This result shows the effectiveness of distribution policies when they are accompanied by advice and information.

Keywords: Heckman procedure, sample selection bias, Frontiers Prevention Project, access, female sexual workers, condoms.

\section{INTRODUCCIÓN}

En muchos países en desarrollo las infecciones de transmisión sexual (ITS) son un problema importante de salud pública. Esto lleva a la implementación de programas públicos que detengan o reduzcan su propagación. Existen múltiples opciones de programas dependiendo de la población objetivo y los recursos físicos e institucionales. Un grupo de estas medidas es la distribución gratuita de condones junto con consejería y la promoción de la prueba de detección del VIH en trabajadoras sexuales (TS).

Existen estudios acerca de los factores que motivan el uso de condones en las TS. Se ha encontrado que los bajos niveles educativos, y mientras más joven se comience el trabajo sexual y se tenga el primer encuentro sexual, exponen a las TS al uso inconsistente de condones. ${ }^{1,2}$ Sin embargo, aquellas TS que reciben gratuitamente condones de parte de funcionarios del sector de la salud o que los compran en los sitios de trabajo, y son asesoradas sobre las pruebas de VIH, exhiben un uso consistente de condones con los clientes ${ }^{3-6}$; lo cual influye en la probabilidad de ocurrencia de alguna ITS según el número y el tipo de compañeros sexuales (clientes o parejas casuales o de largo plazo) 7-11. En relación a la distribución gratuita de condones, existen riesgos de que estos programas no sean exitosos, o sean de eficacia temporal, debido a los bajos niveles de educación, el uso de drogas y alcohol, y la dis- posición de los clientes a pagar tarifas más altas por no utilizar condones. ${ }^{12}$ Más aún, se sabe que existen diferencias significativas en la capacidad de las TS para negociar el uso del condón con los clientes debido a la dependencia económica y la falta de otras fuentes de ingresos. ${ }^{1,6,13,14}$

Sin embargo, son escasos los estudios acerca de los efectos de los programas de distribución gratuita de condones sobre el comportamiento de compra. Más aún, en Ecuador, donde el trabajo sexual está legalizado, y en otros países, estos programas de distribución son permanentes y parte integral de las políticas de control de ITS. De allí, entonces, surge la necesidad de estudios en esta área. Por tanto, el objetivo de este estudio es determinar si la distribución gratuita de condones a las TS afecta la compra de los mismos y su uso consecuente. Esto sería una indicación de la efectividad de estos programas en la promoción de comportamientos sexuales más seguros.

\section{MATERIALES Y MÉTODOS}

\section{Datos}

Los datos corresponden a las encuestas realizadas por el PPF en Ecuador en el 2006, donde 2.566 TS fueron parte de dicho programa. Los datos se recogieron en los sitios de trabajo de las TS en la ciudades de Esmeraldas, Santo Domingo, Quevedo, Machala, Milagro, Daule, Quito y Guayaquil. ${ }^{15}$ 
Para estimar el tamaño de la población a estudiar se realizaron mapeos en cada una de las ciudades con la participación de TS que eran miembros de Organizaciones $\mathrm{No}$ Gubernamentales (ONG). El tamaño muestral se determinó de acuerdo al número mínimo de TS requeridas para detectar una diferencia en el uso del condón desde el último encuentro sexual entre las ciudades intervenidas y las de comparación (véase Tabla 1), considerando los efectos de diseño de la selección por conglomerados (ciudades). Se calculó una diferencia de 10 puntos porcentuales en el uso del condón con un efecto de diseño de 2.2. ${ }^{15}$ Para una descripción más detallada del PPF, sus fundamentos conceptuales y condiciones muestrales de implementación, véanse las referencias ${ }^{15-17}$.

Los cuestionarios recogieron variables sociodemográficas (edad, educación, situación laboral, activos económicos, entre otras), de comportamiento sexual (tipo de parejas sexuales o clientes, prácticas sexuales, uso de condones, tarifas cobradas por el trabajo sexual y detalles sobre los últimos tres clientes), información sobre la pareja estable, conocimiento sobre ITS, y las actitudes hacia personas con VIH. También se incluían preguntas sobre la cantidad de condones comprados y recibidos de forma gratuita, además del gasto en ellos en las cuatro semanas anteriores a la entrevista.

Las TS se clasificaban de la siguiente manera: i) aquellas que trabajaban en la calle; ii) aquellas que trabajaban en burdeles o prostíbulos; y iii) aquellas trabajando en otros sitios. El cuestionario no permite separar aquellas TS que trabajan a través de internet $u$ otros sitios diferentes.

\section{Análisis estadístico}

Para identificar los determinantes de la compra de condones bastaría usar el método de mínimos cuadrados ordinarios (MCO), donde la variable dependiente sería la cantidad de condones comprados. El método de MCO asume que el término residual no incluye alguna variable omitida $u$ otro factor no observable que tenga un efecto significativo sobre la cantidad comprada de condones. Es decir, para obtener estimadores no sesgados a través del análisis de regresión, no debe existir correlación entre el residuo y cualquiera de las variables explicativas ${ }^{18}$. Para ello, el diseño del estudio debe ser completamente aleatorio, de tal manera que las TS no puedan influir en la probabilidad de ser parte de las intervenciones del PPF y de recibir condones gratis. Sin embargo, existen situaciones que podrían afectar esta probabilidad. Primero, una TS podría estar dispuesta a participar en el PPF por las expectativas de recibir condones gratis y realizar ahorros. Segundo, y en sentido contrario, la TS podría negarse a participar debido a una probable prueba de VIH que resulte positiva y la excluya del trabajo sexual. Dado el requerimiento legal de ser VIH negativa para ser TS, esto supondría perder su mayor (y a veces única) fuente de ingresos. En cualquiera de los casos, la TS toma esta decisión sin comunicar al investigador, por lo cual son factores inobservables no expresados a través de las variables explicativas, sino en el término residual.

Además, a priori no es conveniente asumir que el acceso a los programas de distribución de condones es aleatorio ya que existirá un grupo de TS que no es de fácil contacto a causa de la dispersión de sus sitios de trabajo en barrios marginales o en zonas rurales, o por trabajar a través de internet, por ejemplo. Sin embargo, en términos del perfil socioeconómico, estas TS son comparables a aquellas que son parte de la muestra y su omisión afecta la representatividad de la muestra seleccionada. De allí que es probable la existencia de sesgos de selección muestral, los cuales toman la forma de errores de especificación y afectan la ausencia de sesgo de las estimaciones por MCO. ${ }^{19}$ Para ello utilizamos el proceso de Heckman, el cual se compone de dos etapas. En la primera se estima un modelo Probit para la decisión de comprar condones, donde la variable dependiente es una dicotómica que toma el valor de 1 si la TS decide comprar y $O$ en caso contrario. En la segunda etapa se estima un modelo de MCO, donde la variable dependiente es el logaritmo de la cantidad de condones comprados. En esta etapa se incluye el inverso del ratio de Mills estimado en 
el modelo Probit, con el fin de corregir los problemas debidos al sesgo de selección ${ }^{19-21}$.

\section{Variables explicativas para la decisión de comprar (Primera Etapa)}

Para lograr identificación formal, las mismas variables explicativas deben aparecer en ambas etapas del proceso de Heckman; y, al menos una variable que afecte significativamente la probabilidad de compra, no debe aparecer en la ecuación de la cantidad comprada. Estas variables se conocen como las restricciones de exclusión y sirven para corregir el sesgo de selección muestral ${ }^{19,21}$.

Asumimos que de las variables recogidas en el cuestionario, las siguientes sirven como restricciones de exclusión:

i. En Ecuador, si una TS se realiza la prueba del VIH en alguna institución pública de salud, suele recibir condones gratuitamente además de consejería sobre el VIH/ SIDA. Sin embargo, no todas las TS tienen el mismo acceso al sistema de salud pública ni tampoco la misma facilidad de ser contactadas por los funcionarios de salud. De allí que una de las restricciones de exclusión se refiere a si la TS alguna vez se ha realizado esta prueba.

ii. Si la TS ha recibido tratamiento por alguna ITS (diferente del VIH/SIDA), se espera que haya recibido también consejería y asesoría en el cuidado de la salud. Esto constituye una experiencia de aprendizaje, la cual supone una mayor probabilidad de comprar condones con el fin de evitar la recurrencia de ITS. De allí que otra restricción de exclusión sea si la TS ha sufrido de ITS en el pasado.

iii. Las características propias del hogar de las TS pueden explicar el costo de una probable infección por VIH. ${ }^{19,}{ }^{22}$ Es decir, si una TS tuviera que dejar de trabajar, esto implicaría la pérdida de su principal fuente de ingresos. Dado que la mayor parte de la muestra no informa una fuente secundaria de ingresos, esto llevaría a la TS y su familia a situaciones económicas desfavorables. Por tanto, para representar esta situación utilizamos el número de depen- dientes (niños y adultos) que viven en el mismo hogar con la TS. ${ }^{3}$

iv. Una barrera para el acceso a condones es si la familia acepta o tiene conocimiento de que uno de sus miembros es TS. ${ }^{5,23,24}$ Esto afectaría la red de apoyo que refuerce la compra y uso de condones. De allí que otra restricción de exclusión sea si la familia de la TS conoce la naturaleza de su trabajo.

v. Si una TS ha experimentado una rotura del condón, esto significaría un efecto negativo sobre la confianza en ellos como una medida preventiva, lo cual implicaría una menor disposición a comprarlos. Entonces, se utiliza también como restricción de exclusión si la TS ha sufrido alguna vez la rotura de condones con un cliente.

vi. Se utiliza también el uso consistente de condones con los últimos tres clientes. Esto reflejaría la importancia atribuida al condón como parte del trabajo sexual.

\section{Variables explicativas para la cantidad de condones comprados (Segunda Etapa)}

Algunas de las variables explicativas son: (i) precio pagado por cada condón, (ii) ingreso mensual por trabajo sexual y otras fuentes; (iii) ciudad donde trabaja la TS; (iii) estado civil; (iv) tipo de TS según el sitio de trabajo, (v) tarifa promedio con los últimos tres clientes, (vi) nivel de educación, (vii) número de años como TS, (viii) número de clientes pagados; y, (ix) número de condones gratuitos recibidos. Se incluye además el inverso del ratio de mills estimado en la primera etapa, lo cual permite evaluar la existencia del sesgo de selección.

\section{RESULTADOS}

La Tabla 1 muestra la distribución de las TS por ciudades. Las ciudades no intervenidas por el PPF se refieren a aquellas donde las políticas de salud fueron iniciativas del Estado o de ONG; mientras que las ciudades intervenidas son aquellas donde a las políticas se les añadió el Proyecto de Prevención en Fronteras. Los datos de Quito y Guayaquil fueron recogidos como referencias adicionales ${ }^{15}$. 
Las TS que trabajan en prostíbulos componen el $70 \%$ de la muestra, el 17\% informó que trabaja en las calles y el resto se considera en la categoría de "otros". El 8,7\% de las TS reportó estar casada y $40.47 \%$ en unión libre. Por otro lado, el $65 \%$ informó que vive con entre 2 y 6 personas como dependientes.

La Tabla 2 muestra estadísticas descriptivas de la muestra completa (2566 TS). La edad promedio de las TS es de 28 años. La última vez que la TS compró condones gastó, en promedio 5,5 dólares (diferente de cero al $95 \%$ de confianza). En promedio, cada compra fue de 59 condones, donde las TS que trabajan en prostíbulos compraron 65 condones, las que trabajan en la calle 44, y aquellas en la categoría de “otros", 67. En promedio el costo de cada condón fue de 9.4 centavos de dólar. La última compra de condones fue en promedio 9,4 días antes de la encuesta.

Tabla 1. Distribución de las TS por ciudades

\begin{tabular}{llcc}
\hline & & $\mathrm{n}$ & $\%$ \\
\hline \multirow{2}{*}{ Ciudades intervenidas } & Machala & 250 & 9.74 \\
& Milagro & 308 & 12.00 \\
& Daule & 272 & 10.60 \\
\hline \multirow{2}{*}{ Ciudades no interveni- } & Esmeraldas & 287 & 11.18 \\
das por el PPF & Santo Domingo & 254 & 9.90 \\
& Quevedo & 389 & 15.16 \\
& Quito & 399 & 15.55 \\
& Guayaquil & 407 & 15.86 \\
\hline Total & & 2566 & 100 \\
\hline
\end{tabular}

Independientemente del sitio de trabajo, las TS recibieron gratuitamente 8 condones, ya sea de ONG, iniciativas del PPF o agencias del gobierno. Además, el $92 \%$ reportó haber usado condón con cada uno de los últimos tres clientes. De la muestra, el 69\% de las TS experimentó alguna vez la rotura del condón con algún cliente.

En los siete días antes de la encuesta, en promedio, las TS atendieron a 29 clientes y cobraron 9,48 dólares. Esto representa un ingreso mensual de 712 dólares basado solo en el trabajo sexual. El tiempo promedio que la TS pasó con un cliente es de 25 minutos. La mediana de los años como trabajadora sexual es de 3.

De la muestra, un 7\% informó haber participado en algún grupo de apoyo; el 44\% reportó haber recibido tratamiento médico por ITS; y, en el 49,76\% de las TS, sus familias no conocen la naturaleza de su trabajo. El 10\% de las TS que trabajan en la calle informó que nunca habían tenido un examen de VIH, mientras que en aquellas que trabajan en burdeles y otros sitios, esta cifra disminuye al $4 \%$ y $5 \%$, respectivamente.

\section{Análisis de Regresión}

Todas las interpretaciones del análisis de regresión se realizan dentro del $95 \%$ de confianza. La Tabla 3 presenta los efectos marginales de cada variable explicativa sobre la probabilidad de que una TS comprará condones. Se encontraron problemas de datos perdidos en 798 TS, particularmente en las

Tabla 2. Estadísticos Descriptivos de las TS

\begin{tabular}{lcc}
\hline & Promedio & Desviación Estándar \\
\hline Edad & $27.69^{*}$ & 7.75 \\
Gasto en condones la última vez que compró & $5.54^{*}$ & 14.21 \\
Cantidad de condones comprados la última vez & $58.93^{*}$ & 88.2 \\
Cantidad de condones recibidos gratis & $8.43^{*}$ & 9.54 \\
Ingreso mensual & $711.98^{*}$ & 318.42 \\
Número de clientes pagados (últimos 7 días) & $29.27^{*}$ & 30.86 \\
Tarifa promedio con los últimos 3 clientes & $9.48^{*}$ & 12.56 \\
Tiempo promedio con los tres últimos clientes (horas) & $0.42^{*}$ & 1.28 \\
\hline valos calculados sobre 1768 observaciones válidas. *Estadísticamente diferente de cero al 95\% de confianza.
\end{tabular}


variables de precios y cantidad de condones comprados, entre otras relacionadas a cuestiones socioeconómicas y demográficas. De allí que se utilizan 1768 observaciones para el análisis de regresión.

Las TS que trabajan en Esmeraldas, Santo Domingo, Quito y Guayaquil muestran una probabilidad menor de compra en comparación con aquellas que trabajan en Machala. Las TS que trabajan en burdeles muestran una probabilidad mayor hacia la compra en comparación a las que trabajan en la calle. Aunque pequeño, este es un resultado esperado ya que el primer grupo se expone con mayor frecuencia a los programas de distribución. Asimismo, sobre la probabilidad de compra también existe un efecto negativo aunque pequeño, de la tarifa promedio cobrada por la TS. En relación a las restricciones de exclusión, la probabilidad de comprar condones aumenta si la TS alguna vez se ha hecho la prueba del VIH en el pasado, si ha usado consistentemente condones con los últimos tres clientes y si la familia de la TS conoce la naturaleza de su trabajo. No se encuentran efectos significativos a partir de si la TS participa de algún grupo de apoyo y si ha experimentado alguna vez la rotura del condón.

La prueba de Wald rechaza la hipótesis de ausencia de sesgo de selección. De los signos de y (componentes del ratio de Mills), el sesgo de selección motivaría un sesgo hacia arriba si acaso este ratio fuera omitido del análisis de regresión.

En la Tabla 4 se tiene que la elasticidad precio de la demanda de condones es de -0,58. Es decir, por cada aumento porcentual en el precio de los condones, la cantidad comprada disminuye en $0,58 \%$. En relación al objetivo de este artículo, por cada condón que la TS reciba gratuitamente se da un incremento del $1,89 \%$ en la cantidad de condones comprados. Por ejemplo, el promedio de condones comprados pasaría de 58.93 a 60 , es decir, por cada condón recibido gratuitamente, la TS también compraría uno más. Este resul-

Tabla 3. Modelo Probit para la decisión de comprar condones (No: 0, Sí: 1)

\begin{tabular}{|c|c|c|c|}
\hline & & Efecto Marginal & Error Estándar \\
\hline \multirow{7}{*}{ Ciudad: Machala como referencia } & Milagro & -0.118 & 0.337 \\
\hline & Daule & -0.109 & 0.321 \\
\hline & Esmeraldas & $-0.614 \% *$ & 0.301 \\
\hline & Santo Domingo & $-0.780 * * *$ & 0.294 \\
\hline & Quevedo & -0.346 & 0.286 \\
\hline & Quito & $-0.976 * * *$ & 0.275 \\
\hline & Guayaquil & $-0.666 * *$ & 0.283 \\
\hline \multirow{2}{*}{$\begin{array}{l}\text { Tipo de TS: trabaja en calle como } \\
\text { referencia }\end{array}$} & Burdel & $0.219 *$ & 0.133 \\
\hline & Otro & -0.061 & 0.162 \\
\hline \multicolumn{2}{|c|}{ Tarifa promedio con los últimos tres clientes } & $-0.007 *$ & 0.004 \\
\hline \multicolumn{2}{|c|}{ Ha sido examinada por VIH alguna vez (Sí: 1 , No: 0) } & $0.619 * * *$ & 0.184 \\
\hline \multicolumn{2}{|c|}{ Familia conoce que es TS (Sí: 1, No: 0) } & $0.227 * *$ & 0.105 \\
\hline \multicolumn{2}{|c|}{ Uso condón con 3 últimos clientes (Sí: 1, No: 0) } & $0.452 *$ & 0.187 \\
\hline \multicolumn{2}{|l|}{ Intercepto } & $0.989 *$ & 0.465 \\
\hline \multicolumn{2}{|l|}{$\rho$} & $-0.238 * *$ & 0.120 \\
\hline \multicolumn{2}{|l|}{$\ln (\sigma)$} & $-0.0793 * * *$ & 0.018 \\
\hline \multicolumn{2}{|l|}{ Número de observaciones } & 1768 & \\
\hline \multicolumn{2}{|l|}{ Observaciones censuradas } & 127 & \\
\hline \multicolumn{2}{|l|}{ Observaciones no censuradas } & 1641 & \\
\hline
\end{tabular}


Tabla 4. Modelo MCO para el logaritmo de la cantidad de condones comprados

\begin{tabular}{|c|c|c|c|}
\hline & & Coeficiente & Error estándar \\
\hline \multicolumn{2}{|l|}{ Logaritmo del precio } & $-0.581 * * *$ & 0.032 \\
\hline \multicolumn{2}{|l|}{ Ingreso mensual } & $0,00013 \cdots *$ & $5.29 \mathrm{E}-05$ \\
\hline \multirow{7}{*}{ Ciudad: Machala como referencia } & Milagro & $-0.211 * \%$ & 0.093 \\
\hline & Daule & $-0.463 * \%$ & 0.081 \\
\hline & Esmeraldas & $-0.513 * * *$ & 0.104 \\
\hline & Santo Domingo & $-0.287 * * *$ & 0.105 \\
\hline & Quevedo & $-0.405 \% * \%$ & 0.075 \\
\hline & Quito & $-0.545 \% *$ & 0.096 \\
\hline & Guayaquil & -0.0828 & 0.081 \\
\hline \multirow{2}{*}{ Tipo de TS: trabaja en la calle como referencia } & Burdel & $0.129 *$ & 0.068 \\
\hline & Otro & 0.0541 & 0.095 \\
\hline \multirow{2}{*}{$\begin{array}{l}\text { El nivel de educación alcanzado: escuela primaria } \\
\text { como referencia }\end{array}$} & Secundaria & $0.0931 *$ & 0.050 \\
\hline & Otro & 0.0717 & 0.083 \\
\hline \multicolumn{2}{|l|}{ Tiempo como TS (años) } & $-0.0113 *$ & 0.006 \\
\hline \multirow{2}{*}{ Estado civil: casada/unión libre como referencia } & Separada/Divorciada/Viuda & -0.0388 & 0.095 \\
\hline & Soltera & $-0.0893 *$ & 0.047 \\
\hline \multicolumn{2}{|l|}{ Número de clientes pagados } & $0,00103 \cdots *$ & 0.0003 \\
\hline \multicolumn{2}{|l|}{ Número de condones gratuitos recibidos la última vez } & $0.0188 * * *$ & 0.002 \\
\hline \multicolumn{2}{|l|}{ Intercepto } & $1.838 * * *$ & 0.149 \\
\hline
\end{tabular}

Notas: $* * \mathrm{p}<0.01, * * \mathrm{p}<0.05, * \mathrm{p}<0.1$. Prueba Wald de ecuaciones independientes $(=0)$ : chi-cuadrado(1)=3.93, Valor P: 0.0475 . Observaciones no censuradas: 1641 . Observaciones censuradas: 127.

tado refuerza los objetivos de las políticas de distribución y asesoramiento en Ecuador, es decir, la distribución gratuita de condones no implica que las TS dejen de comprarlo. Más aún, por cada 100 dólares de aumento en el ingreso mensual, la compra de condones también aumenta en un $1,35 \%$.

Si una TS ha completado al menos un año de educación secundaria, la cantidad de condones comprados es $9 \%$ más que aquellas que completaron solo la primaria. Comparadas con Machala, en el resto de ciudades, a excepción de Guayaquil, la compra de condones en promedio es menor entre $21 \%$ y $60 \%$. Además, las TS que trabajan en burdeles compran $14 \%$ más condones en comparación a las que trabajan en la calle. Las TS que son solteras compran casi $9 \%$ menos que aquellas que reportan estar casadas o en unión libre. Finalmente, por cada año que una mujer sea TS, la compra se reduce en un $1,13 \%$, y por cada cliente adicional, la compra aumenta en $0,1 \%$.

\section{DISCUSIÓN}

En este estudio se analiza el comportamiento de compra de condones de las TS en Ecuador, tomando como caso de estudio las encuestas del Proyecto de Prevención en Fronteras. Se evalúa cómo la distribución gratuita de condones afecta la compra y su uso consecuente. Debido a la no aleatoriedad en el acceso a estos programas, utilizamos un proceso de Heckman ${ }^{19,20}$ e identificamos los determinantes tanto de la probabilidad de comprar condones como de la cantidad comprada. Los resultados comprueban que existe sesgo de selección muestral, lo cual justifica el proceso de Heckman por encima de MCO.

Se encuentra que la distribución gratuita de condones implica un efecto positivo en la compra de los mismos. Este resultado se explica por el componente de consejería y asesoramiento que acompañan los programas de distribución, sin importar que sean iniciados por el PPF o 
autoridades públicas de salud. Junto con el uso consistente con todos los clientes y el apoyo del núcleo familiar. Por tanto, los programas de distribución, para la evidencia disponible, son efectivos en la promoción de medidas de protección contra las ITS.

Sin embargo, existen algunas condiciones a considerar en el diseño de estos programas. Los resultados para la probabilidad de compra muestran que existen indicios de que, debido a la pobreza o dependencia económica, una TS estaría dispuesta a asumir el riesgo de contraer alguna ITS al permitir al cliente no usar condones, dado que este riesgo adicional se reflejaría en una prima por encima de la tarifa promedio..$^{25}$ Asimismo, los resultados también muestran que la competencia con TS más jóvenes incentivaría a TS de mayor edad, o con mayor tiempo como TS, a obviar el uso del condón con algunos de los clientes.

Algunas limitaciones del estudio que sirven de base para investigación futura son las siguientes: i) no se considera la calidad de los condones o las condiciones en que se almacenan; ii) dado el carácter transversal del estudio, no es posible identificar relaciones de causalidad; sin embargo, la dirección de la asociación entre las variables explicativas y la cantidad de condones comprados puede ser fundamentada en el contexto social. iii) Las restricciones de exclusión podrían relacionarse con las condiciones sociales que motivan a una mujer a iniciarse como TS, por ejemplo, abuso familiar a temprana edad, secuestro y extorsión de la TS o su familia, o ser víctima de redes de tráfico de personas.

\section{AGRADECIMIENTOS}

Se agradece a Amira Herdoíza, Pike Brown, W. Douglas Shaw, Richard Dunn y María Auxiliadora Olivares.

\section{REFERENCIAS}

1. Solomon MM, Smith MJ, Del Rio C. Low educational level: a risk factor for sexually transmitted infections among commercial sex workers in Quito, Ecuador. Int J STD AIDS. 2008 Apr.; 19(4): 264-7.

2. Lippman S a, Donini A, Díaz J, Chinaglia M, Reingold A, Kerrigan D. Social-environmental factors and protective sexual behavior among sex workers: the Encontros intervention in Brazil. Am J Public Health. 2010 Apr.; 1: 100(1): S216-23. Disponible en:

3. Oladosu M. Consistent condom use dynamics among sex workers in Central America: 1997-2000. J Biosoc Sci. 2005 Mar. 7; 37(04): 435-57.

4. Kayembe PK, Mapatano M a, Busangu a F, Nyandwe JK, Musema GM, Kibungu JP, et al. Determinants of consistent condom use among female commercial sex workers in the Democratic Republic of Congo: implications for interventions. Sex Transm Infect. 2008 Jun.; 84(3): 202-6.

5. Shannon K, Strathdee $S$ a, Shoveller J, Rusch M, Kerr T, Tyndall MW. Structural and environmental barriers to condom use negotiation with clients among female sex workers: implications for HIV-prevention strategies and policy. Am J Public Health. 2009 Apr.; 99(4): 659.65.

6. De la Torre A, Havenner A, Adams K, Ng J. Premium sex: Factors influencing the negotiated price of unprotected sex by female sex workers in Mexico. J Appl Econ. Universidad del CEMA. 2010 May; 13(1): 67-90.

7. Macaluso M, Demand MJ, Artz LM, Hook EW. Partner type and condom use. AIDS. 2000 Mar. 31; 14(5): 537-46.

8. Outwater A, Nkya L, Lwihula G, O'Connor P, Leshabari M, Nguma J, et al. Patterns of partnership and condom use in two communities of female sex workers in Tanzania. J Assoc Nurses AIDS Care. 2000; 11(4): 46-54.

9. Allen B, Cruz-Valdez A. Afecto, besos y condones: el $\mathrm{ABC}$ de las prácticas sexuales de las trabajadoras sexuales de la Ciudad de México. Salud Pública de Méx [en línea]. 2003 [consultado 2.05.2014]; 594-607. Disponible en: http://scielo.unam.mx/scielo.php?pid=S0036$36342003001100004 \&$ script $=$ sci_arttext

10. Stephenson R, Beke A, Tshibangu D. Contextual influences on contraceptive use in 
the Eastern Cape, South Africa. Health Place. 2008 Dec.; 14(4):841-52.

11. Eaton L a, Kalichman SC, Cherry C. Sexual partner selection and HIV risk reduction among Black and White men who have sex with men. Am J Public Health [en línea]. 2010 Mar. [consultado 2.05.2014]; 100(3): 503-9. Disponible en: http://www.pubmedcentral.nih.gov/articlerender.fcgi?artid $=2820$ 059\&tool $=$ pmcentre $z$ \&rendertype $=$ abstract

12. Ntumbanzondo M, Dubrow R, Niccolai LM, Mwandagalirwa K, Merson MH. Unprotected intercourse for extra money among commercial sex workers in Kinshasa, Democratic Republic of Congo. AIDS Care. 2006 Oct.; 18(7): 777-85.

13. States U, Deb P, Grossman M, Haider S, Kaestner R, Laffont J, et al. HIV breakthroughs and risky sexual behavior: the diffusion of breakthrough HIV drugs in developed coun- tries has transformed the disease from a death sentence into a manageable chronic condition. Since 1995, the overall U.S. AIDS death rate has fal. 2006; (August).

14. Stoebenau K, Hindin MJ, Nathanson C a, Rakotoarison PG, Razafintsalama V. “...But then he became my sipa": the implications of relationship fluidity for condom use among women sex workers in Antananarivo, Madagascar. Am J Public Health [en línea]. 2009 May [consultado 2.05.2014]; 99(5): 811-9. Disponible en: http://www.pubmedcentral. nih.gov/articlerender.fcgi? artid $=2667855 \& \mathrm{t}$ ool $=$ pmcentre $\&$ rendertype $=$ abstract

15. Gutiérrez JP, Atienzo EE, Bertozzi SM, McPherson S. Effects of the Frontiers Prevention Project in Ecuador on sexual behaviours and sexually transmitted infections amongst men who have sex with men and female sex workers: challenges on evaluating complex interventions. J Dev Eff. Routledge; 2013 Jun http://dx.doi.org/10.1080/1943 9342.2013.780088

16. Gutiérrez JP, Econ M, Sal D, Molina-Yépez D, Esp M, Samuels F, et al. Uso inconsisten- te del condón entre trabajadoras sexuales en Ecuador: resultados de una encuesta de comportamientos. 2006; 48(2): 104-13.

17. Gutierrez J-P, McPherson S, Fakoya A, Matheou A, Bertozzi SM. Communitybased prevention leads to an increase in condom use and a reduction in sexually transmitted infections (STIs) among men who have sex with men (MSM) and female sex workers (FSW): the Frontiers Prevention Project (FPP) evaluation results. BMC Public Health [en línea]. 2010 Jan.; 10: 497. Disponible en: http://www.pubmedcentral. nih.gov/articlerender.fcgi artid=2940912\&t ool=pmcentrez\&rendertype $=$ abstract

18. Wooldridge JM. Econometric Analysis of Cross Section and Panel Data [en línea]. MIT Press; 2010. Disponible en:

19. Heckman J. Sample selection bias as a specification error. Econom J Econom Soc. 1979; 47(1): 153-61.

20. Bushway S, Johnson BD, Slocum LA. Is the Magic Still There? The Use of the Heckman Two-Step Correction for Selection Bias in Criminology. J Quant Criminol. 2007 Mar 23; 23(2): 151-78.

21. Puhani P. The Heckman Correction for Sample Selection and Its Critique. J Econ Surv. 2000 Feb.; 14(1): 53-68. Disponible en: $\quad$ http://doi.wiley.com/10.1111/14676419.00104

22. Nawata K. Estimation of sample selection bias models by the maximum likelihood estimator and Heckman's two-step estimator. Econ Lett. 1994 May; 45(1): 33-40.

23. Koenig $M$ a, Hossain MB, Whittaker M. The influence of quality of care upon contraceptive use in rural Bangladesh. Stud Fam Plann. 1997 Dec.; 28(4): 278-89.

24. Benefo KD. Determinants of condom use in Zambia: a multilevel analysis. Stud Fam Plann. 2010 Mar.; 41(1): 19-30.

25. Arunachalam R, Shah M. Compensated for Life: Sex Work and Disease Risk. J Hum Resour. 2013 Apr. 1; 48(2): 345-69. 